\title{
Implementation of the School Administration and Management System: a Hong Kong experience
}

\author{
B.C.H. Ip Tsang and S.Y.F. Lee \\ 23/F, Wu Chung House, Wanchai, Hong Kong \\ Fax: (852)-25758952
}

\begin{abstract}
In September 1993, the Hong Kong Government allocated a sum of HK $\$ 570$ million (US\$73 million) for the implementation of a five-year Information Systems Strategic Plan in the Education Department. About forty percent of the sum is for the School Administration and Management System (SAMS) project. This paper aims at sharing the experience of implementing that project in Hong Kong schools. Extensive user participation and carefully planned implementation tasks are the key elements in the SAMS project. The paper describes the mode and the extent of user participation and the major implementatation tasks required. Management issues arising from the implementation of SAMS are highlighted and the way forward in the management of the project is proposed.
\end{abstract}

\section{Keywords}

Educational management, government, information technology

\section{INTRODUCTION}

In 1990 the Hong Kong Government Public Sector Reform Study Team recommended that the Education Department (ED) should obtain expert help to identify the information needs of the Schools Education Programme and develop appropriate management information systems. A consultancy study was then conducted and a five-year Information Systems Strategic Plan (ISSP) was proposed. The ISSP covered two parts: Corporate Systems for the ED and SAMS for the schools.

In September 1993 the Hong Kong Government allocated a sum of HK \$570 million (US\$73 million) for the implementation of the ISSP in the ED within a five-year period, about 40 percent of which was for the SAMS project. The SAMS project aims to provide an integrated computerised system to schools for supporting the major management and administrative processes, and for transmitting electronically 
information on students, teachers and schools between schools and ED. The SAMS consists of twelve core applications and four supporting applications.

The SAMS project adopts an in-house development approach in which the Information Technology Services Department (ITSD) of the Hong Kong Government acts as the developer. Two major methodologies have been used for the project management and system development. They are the methodology of Projects In Controlled Environment (PRINCE) for the project management and the Structured System Analysis and Design Methodology (SSADM) for the development of applications.

The SAMS project is characterised by the following features:

1. Linkage with ED: Schools and ED communicate frequently with each other, through circulars, letters, surveys or forms. Under the ISSP, there is a standardisation of data definitions across the ED Corporate Systems and SAMS. Coupled with the provision of a dial-up telephone line, communication between both parties through electronic media is enabled.

1. Bilingual system design supporting both Chinese and English languages: The Official Language Ordinance of Hong Kong provides that the official languages in Hong Kong are English and Chinese. In practice, for undertaking the administrative tasks, Hong Kong secondary schools usually use both languages while primary schools use Chinese language predominantly. To meet the schools' needs, the SAMS is a bilingual system with the options of Chinese and English screen display. In view of the limited Chinese character set available from Microsoft Chinese Windows, the ED has co-ordinated the creation of an extended Chinese character set with an additional 2,700 Chinese characters.

1. Centrally-developed tailor-made system for 1300 schools: The target users of the SAMS are 1300 schools of various types. The design of the SAMS therefore emphasizes its flexibility to suit the basic needs of the diverse school types (e.g., curriculum, financial modes, number of administrative units made up by different sessions or heads, assessment systems, student information requirements).

1. Pilot roll out and application enhancement: Piloting is adopted for testing the users' receptiveness to the system and for identifying areas for fine-tuning. In SAMS version 1.0 ten schools of different nature were selected as pilot schools. The lead time for the actual rollout after the piloting was two months, which however proved to be inadequate for any crucial fine-tuning to be made. Based on that experience, in the subsequent rollout, the piloting period has been extended. Concerning the application enhancement, there are various channels through which users may give suggestions on the design and functionality of SAMS for consideration in the future version of SAMS that are released on a half-yearly basis.

2. Local Area Network (LAN) with Graphical User Interface (GUI) : Regarding the system configuration of the SAMS, in each primary and secondary school, a group of microcomputers will be installed and linked together to form a local area network (LAN) in the school building. The LAN consists of one to two file servers connected to five to nine workstations depending on the total number of operating classes of the school. Together with SAMS, standard software packages on word processing, spreadsheet and database management are provided. Windows NT Server 3.5 is used as the network operating system. The system is developed under a Windows environment with Graphical User Interface (GUI), allowing users to communicate with SAMS by using a mouse. 
3. Provision of End-user Computing tool: At the time of the selection of the development tool, FoxPro could fulfill the requirement of running under Windows. It had the required GUI; was capable of efficient database management, and was considered the most appropriate tool for users at the time. Furthermore, FoxPro for DOS had already been in use in schools at the time when the SAMS project was initiated. As the users had already been trained in the use of the FoxPro, they have little difficulty in moving to FoxPro for Windows. The same software is also taught in the secondary school curriculum and is very popular among school teachers, which helps pave the way for the development of end-user computing in SAMS schools.

4. Keep abreast of the latest technology: The Hong Kong Government has been providing both hardware and software enhancement to this centrally developed system. The equipment supplies contracts are periodically renewed to ensure that the project can benefit from the latest technology. For example, the workstations currently provided to schools are a $80486 \mathrm{DX} 4100 \mathrm{Mhz}$ system instead of the $8048633 \mathrm{Mhz}$ system provided in the early stage. Additional hardware such as the LaserJet 4 Plus printers and Chinese writing pads are further provided on top of the standard configuration. Besides, implications of deploying new software to schools are always studied and new releases of software are supplied if needed. Currently the impact of using Chinese Windows 95 in SAMS is being explored.

\section{QUESTIONS TO BE ANSWERED}

In the following sections, we would like to share our experience in the implementation of SAMS in the public sector schools in Hong Kong by answering the three questions stated below:

(a) What are the essential tasks involved in the development and execution of this centrally developed school management information system?

(b) What are the implementation issues to be addressed and how to manage the issues?

(c) What is the way forward for this system?

\section{ANSWERS TO QUESTIONS STATED}

In providing answers for question (a) above, the modes of user participation and other major tasks will be analysed; for question (b), the various issues will be identified and the method of management analysed; for the last question, a plan for the on-going development of the System will be proposed.

\subsection{User Participation}

Throughout the development of the SAMS, user involvement is extensive - on both regular and need basis. 
Teacher Secondment - Under the scheme of teacher secondment, teachers with school administration and IT skills are seconded to work full-time in the ED on a one-year tenure. Since the commencement of this secondment system in September 1994, about 30 heads and teachers from different types of schools have worked jointly with the ED staff in the SAMS project team. As the nature and requirements of the 1300 target schools are diverse, it is neither cost-effective nor operationally feasible to interview all schools to collate their requirements. Being the end-users of the SAMS, the seconded teachers give comments and suggestions during all stages of system development and project implementation. They also collate requirements from the co-workers in schools, assist in the design of test plans, provide test data and conduct user acceptance tests.

Advisors - There is an advisor system, through which heads, teachers or school clerks are performing an advisory role in specifying user requirements during the feasibility studies, system analysis and design and implementation stages. The advisors play an important role in advising on the priorities set for those diverse requirements. Since the kick-off of the SAMS project, about 20 heads, teachers and clerks have been serving as part-time advisors to the SAMS project.

System Implementation Co-ordinators - There are also experienced SAMS teacher users serving as part-time system implementation co-ordinators. They perform the dual roles of suggesting the appropriate functions to be included in SAMS and serving as the regional co-ordinator to offer on-site advice and support to schools in the neighbourhood. At present, there are about 20 teachers serving in this capacity.

ED Officers - Working alongside the above users are the ED officers who have both teaching and school administration experience. They perform the role of middlemen between the developer (i.e. ITSD) and the end-users. To ensure that the schools' diverse needs are identified, screened, prioritised and properly addressed in the applications, these officers co-ordinate activities like school visits, workshops, check-point meetings, application walkthroughs with the participation of a good representation of schools with different characteristics.

Others - Participants in the above meetings are school representatives from major sponsors, school councils and all types of schools. The number of participants for each meeting ranges from about 5 to 60 . In almost all meetings and various school visits, the ITSD developers are also present to acquire first-hand information from the school users, which facilitates the subsequent information exchange and discussion among ITSD developers and the ED officers. Since the inauguration of the SAMS project in September 1993, over 120 meetings have been organised with the participation of more than 3000 person-sessions from about 20 percent of the 1300 target schools.

\subsection{Other major tasks}

Before the actual operation of the SAMS in schools, the ED identifies resources, plans and co-ordinates a series of tasks. Although all the tasks are relatively simple, the aggregate amount of work, due to the number of schools involved and the pledges to complete all installation tasks for a single school within a short period of time, has made the administrative and co-ordination work very difficult. The major tasks involved are described below: 
1. Site preparation: Schools have to identify suitable sites for the installation of the networked system. After the relevant authority's approval of the network requirement reports, the contractor carries out the site works, including installation of power points, signal cabling and minor builders' works. Delivery, installation and acceptance of hardware and software then follow. Detailed planning, close supervision, frequent communication and better coordination are the keys to the successful installation of the LANs in schools.

1. Data conversion and system uploading: Schools have to convert existing data, in either manual or electronic forms, into the data format of the SAMS. In choosing the methodology for data conversion, the use of optical mark reader or optical character recognition has been ruled out as the former method requires a lot of transcription work for teachers while the latter needs much skill in writing and effort in fixing errors. Conversion can now be done by using a conversion program or filling in coding sheets for data preparation service arranged by the ED.

1. Training: School staff attend training courses on basic IT (covering operating system, spreadsheet, Chinese word processing and database management), SAMS applications (ranging from one to two days for each application) and the related courses like 'management of change' for heads and 'LAN administration' for system administrators. Other courses or seminars with special themes are also organised on a need basis.

1. Outside professional services: To meet the pledge of completing the implementation of SAMS in all 1300 schools within a five-year period, and to minimise impacts on schools resulting from the initial implementation of the SAMS, the ED has been adopting two methods of service acquisition. First, there are some services contracted to outside service providers. The contract services include those for site preparation works, compilation of SAMS procedure manual and training kits, data input tasks and on-site SAMS uploading. Second, allowances are given to teachers who help to perform in-house basic IT training, data conversion and related SAMS implementation tasks.

\subsection{Identification and management of issues arising from SAMS implementation}

With the implementation of the SAMS, there are some related issues that require the ED's close monitoring. In the following paragraphs, the issues are identified and the methods of management are also outlined.

\subsubsection{Core functions in SAMS not satisfying individual needs of schools}

In spite of the efforts made to address the requirements of different types of schools, there are comments from schools suggesting that the core functions provided cannot meet their specific needs. Taking the assessment system as an example, though there is a mechanism built in SAMS for applying different algorithms in mark calculation, among the 1300 target schools, there are some cases that the SAMS cannot handle. In fact, the SAMS has been developed with the objective of providing the core functions essential to assist schools in performing the daily administrative and management tasks. At the beginning of the system studies, it had been noted that with schools' varied characteristics and diverse needs, SAMS may not fully meet the individual requirements 
of each and every school. For addressing this issue, two lines of action have been adopted. First, with user feedback, SAMS is constantly enhanced aiming at enriching and improving the functions. For example, in SAMS version 1.4, released about one and a half years after version 1.0, there are several new functions. The functions provide facilities for the processing of multiple school levels and sessions, different subject groups, special purpose classes and educational programmes. Such inclusions increase the flexibility and functionality provided in the various applications like assessment and reporting. Second, facilities are available to extract data from the SAMS database for further manipulation and processing. An end-user development tool, Microsoft FoxPro $2.5 \mathrm{~b}$ for Windows at present, is provided to schools for developing tailored add-on programmes. Authorised users with programming background can access the databases in read only mode. Data dictionary is also prepared for users. In addition, since the commencement of the SAMS rollout in September 1994, an individual Section has been set up in ED with the mission to promote and co-ordinate add-on programme development by planning, supporting and offering training relating to end-user computing.

\subsubsection{Users' reservation of switching from existing computer systems to SAMS}

In some schools, especially the secondary ones, some systems are already in use, assisting in the administrative tasks. The systems may have been in service for a while and are proved to be functionally effective. The need to switch to another system of SAMS surely causes a certain amount of resistance. In this regard, most schools are adopting an open attitude. In actual fact, the SAMS does have the merit of being a centralised system; teachers familiar with SAMS can carry this knowledge and experience with them when they change from one school to another. As SAMS becomes a common tool among schools, the loss of 'technical hands' is no longer a threat to the smooth school administration. For addressing this concern direct, enhancements are being made to the coming version of SAMS in which 'crossing bridges' will be provided to allow the import of data generated from other computer systems. Data imported to SAMS should be in the pre-defined data format. In this approach, the flexibility for schools in using existing systems for the processing of certain functions not provided under the existing scope of SAMS increases tremendously.

\subsubsection{Varied computer literacy and users' resistance to change}

In some schools, teachers are not ready to use computers to assist in their daily work as it is not their normal practice. To help eliminate their resistance to the use of computers, different types of training in improving their computer literacy are organised. In addition to training in SAMS applications, basic IT training courses are also organised. Training modes are varied in order that more teachers can grasp the required skills. For example, school-based training is planned in which teachers of the same school, mostly computer teachers, may perform the role of trainer giving lessons outside school hours to all teachers of the same school. This reduces the disturbance to the normal lessons and increases the incentives of teachers to attend training session in a familiar environment.

Furthermore, the ED is providing an active supportive service to all schools in using SAMS. Help desks are operated by means of hot-lines, bulletin board services, fax, etc. On-site service is provided and appointment can be made in which officers can stand-by serving schools using the SAMS in performing time-critical tasks like report card printing. Experienced SAMS users are also recruited to serve as part-time system implementation co-ordinators offering on-site coaching to schools with difficulties in using SAMS. Besides, Saturday workshops and talks on special themes are regularly organised to resolve the schools' problems in using SAMS. 


\subsection{The way forward}

Unlike those development tools for workstations or a mainframe platform, the tools for the PC platform of SAMS are cheaper, easier to manage and have more options. Coupled with the good computer literacy among the teachers who have knowledge of the school's daily business, a significant number of users, especially those from secondary schools, have been developing add-on programmes using the SAMS data. The Add-on Programme Development Section of the ED has been set up to formulate strategies on End-User Computing (EUC) and to plan and conduct activities relating to the nurturing of EUC culture among school users. In addition, regional resources centres are planned to be set up for organizing EUC activities including training, development of add-on programmes and trying out of new development tools. Moreover, the regional resource centres provide the meeting ground for school users to share their experience and to provide peer-to-peer support services. In the long run, with the germination of the EUC culture, it is hoped that the school users who know their business needs well and have the ability to develop add-on programmes, will supplement the SAMS to cater for the needs of individual users.

\section{CONCLUSION}

The development and implementation of the SAMS project are due for completion by 1998. The completion of the project does not signify an end to the ED's information system strategy in schools. Instead, in order to keep pace with the new requirements arising from changes in educational policies and the trend of information technology, the SAMS will expand and evolve, with enhancement and maintenance of the core functions on the one hand, and the development of end-user computing activities on the other. It is our vision that the SAMS acts as the backbone of the Hong Kong school administrative and management system, and concurrently, that the users continue to develop add-on programmes to this centralised system, making it more useful and beneficial to users.

\section{BIOGRAPHIES}

Mrs Betty Ip is the Senior Education Officer (Administration) of the Education Department of Hong Kong. She obtained a Bachelor of Science Degree in 1976 and a Master of Education Degree in 1985, both from the University of Hong Kong. She has worked on the application of information technology in education administration and management for fifteen years. In the past three years, she has been responsible for the overall administration of the School Administration and Management System Project.

Mr Steve Lee is the Senior Education Officer (Administration) of the Add-on Programme Development Section of the Education Department of Hong Kong, responsible for the promotion and development of End-user Computing for the Information System Strategy Project. He obtained a Bachelor of Science degree in 1977 and a Master in Business Administration in 1992, both from the Chinese University of Hong Kong. In 1995, he obtained a Master of Science degree in Information Systems from the George Mason University. He has been working as an education administrator for thirteen years of which seven years have been in the information system for education management field. 